\title{
Non-immune Hydrops in Neonates: A Tertiary Care Center Experience
}

\author{
Shamnad Madathil, Pratima Anand, Ashok K Deorari, Ramesh Agarwal, Anu Thukral, M Jeeva Sankar and \\ ANKIT Verma \\ From Division of Neonatology, Department of Pediatrics, All India Institute of Medical Sciences, New Delhi, India.
}

\author{
Correspondence to: Dr Anu Thukral, \\ New Private Ward-1st Floor, All India \\ Institute of Medical Sciences, Ansari \\ Nagar, New Delhi 110029, India. \\ dranuthukral@gmail.com \\ Received: January 28, 2019; \\ Initial review: July 12, 2019; \\ Accepted: November 22, 2019.
}

Objective: To evaluate the clinical profile and outcome of neonates with non-immune hydrops $(\mathrm{NIH})$. Methods: Data of all the $\mathrm{NIH}$ cases admitted to neonatal intensive care unit at our center, New Delhi from January, 2010 to October, 2017 were extracted from hospital records, which included clinical profile and outcomes. Results: Of the 17,299 total births, 27 neonates were identified to have $\mathrm{NIH}$. Antenatal interventions were undertaken in five $(18.5 \%)$ cases. The most common etiology of NIH was cardiac $(n=5 ; 18.5 \%)$. Two babies with chylothorax were successfully managed with octreotide infusions. Overall survival rate of $\mathrm{NIH}$ was $70.3 \%(n=19)$. All neonates with a suspected genetic syndrome died. Conclusion: Multidisciplinary team including obstetricians, pediatric surgeons, geneticists and neonatologists can improve outcome in neonates with $\mathrm{NIH}$.

Keywords: Antenatal diagnosis, Chylothorax, Nonimmune hydrops fetalis.
$\mathrm{H}$ ydrops fetalis (HF) is defined as the presence of extracellular fluid in at least two fetal body compartments or one body compartment with the presence of skin edema ( $>5 \mathrm{~mm}$ thickness) [1]. The incidence of non-immune hydrops (NIH) varies between 1 in 1700 to 3000 live births [2]. Over the years, there has been a trend towards increased incidence of NIH with the advent of Rh-immune globulin for prevention of immune hydrops [3].The reported global incidence of non-immune cases is nearly $90 \%$ of all cases of hydrops fetalis; however, this may not hold true for Indian subcontinent as the coverage of Rh- immune globulin is still not universal [4].

With recent advances in antenatal diagnosis and management, survival rate of $\mathrm{NIH}$ has improved significantly to the tune of $50 \%$. Prognosis depends mainly on the underlying etiology. The present study aims to delineate the etiological profile and evaluate outcome of the neonates with NIH at a tertiary care center in India.

\section{METHODS}

Data of all NIH cases delivered between January, 2010 and October, 2017 was analyzed retrospectively. Diagnosis of NIH was as per standard criteria [1].

Data on demographic variables, antenatal and postnatal course of the fetus and neonates was extracted from case records using standard predesigned proforma. The antenatal work up of NIH at our center includes hematological evaluation, serology for fetal infections, ultrasound doppler to assess fetal anemia, fetal echocardiography and evaluation of ascites and pleural fluid wherever feasible. Delivery room management is carried out by a team, ventilation is supported by T-piece resuscitator for delivery of positive pressure ventilation with higher initial pressures of $20 \mathrm{~cm} \mathrm{H}_{2} \mathrm{O}$ and intubation as per Neonatal resuscitation program (NRP) protocol.

Details of management in the NICU including ventilator support [conventional or high frequency ventilation (HFOV)], use of inotropes, inhaled nitric oxide (iNO), and evidence of persistent pulmonary hypertension of newborn (PPHN) and shock were extracted from the neonatal case records. Interventions such as use of anti-arrhythmics, octreotide infusion and surgery, where applicable were also noted.

Statistical analyses: All the data extracted were compared between babies who survived and expired in hospital. Chi-square test or Fisher exact test, was used for categorical variables and student $t$ test for comparing continuous variables with normal distribution and Mann Whitney U test for skewed data. Univariate followed by multivariate analysis was done to assess the risk factors for mortality. Statistical analysis was done using Stata 13.0 (Stata Corp, College Station, TX).

\section{RESULTS}

There were 27 cases of NIH diagnosed among the 17,299 births during the study period providing an incidence $(95 \% \mathrm{CI})$ of $1.5(1.0-2.2)$ per 1000 total births. There was a 
slight female preponderance $(55.5 \%)$. The mean (SD) birth weight and gestational age was 2315 (543) grams and 33.9 (3.1) weeks, respectively. Majority of them were diagnosed antenatally $(n=26)$. Major clinical features identified were ascites $(n=20)$, skin edema $(n=16)$, pleural effusion $(n=14)$ and pericardial effusion $(n=12)$, which were not mutually exclusive.

Table I provides the etiology of NIH in this study. Of these, etiology was identified antenatally in 12 cases and fetal treatment was undertaken in five of these cases which included pleuro-amniotic shunt $(n=2)$, peritoneoamniotic shunt $(n=1)$ and maternal digitalization/ antiarrythmics $(n=2)$. Delivery room intubation was required in 13 cases, intravascular volume expanders in four and chest compressions with intravenous adrenaline in three cases. Emergency paracentesis and thoracocentesis were required in nine cases and 13 cases had severe birth asphyxia. There were 14 neonates who required mechanical ventilation and ten had shock requiring inotropic support. Three neonates had persistent pulmonary hypertension of newborn (PPHN) requiring inhaled nitric oxide (iNO) support. Other interventions included octreotide infusions $(n=2)$, and surgical exploration for meconium peritonitis $(n=1)$.

Overall survival rate of NIH cases was 70\% $(n=19)$. All three neonates with suspected genetic syndrome died. On univariate analysis, only APGAR score at 1 and 5 minute were found to be statistically significant; median (range) APGAR score at $5 \mathrm{~min}$ in survivors being 8 (6-9) compared to 6(2-6) in those who died.

Table I Etiology of Non-immune Hydrops $(N=27)$

\begin{tabular}{lr}
\hline Diagnosis & No. (\%) \\
\hline Cardiovascular & $5(18.5)$ \\
$\quad$ Arrhythmia (all) & $3(11.1)$ \\
Others & $2(7.4)$ \\
Chylothorax & $4(14.8)$ \\
Suspected genetic syndrome & $3(11.1)$ \\
$\quad$ Down syndrome & $1(3.7)$ \\
Smith Lemli Opitz syndrome & $1(3.7)$ \\
Pena Shokier syndrome & $1(3.7)$ \\
Chorioangioma placenta & $2(7.4)$ \\
Meconium peritonitis & $2(7.4)$ \\
Suspected glycogen storage disease (GSD) & $2(7.4)$ \\
Twin to twin transfusion syndrome (TTTS) & $1(3.7)$ \\
Anemia of unknown cause & $1(3.7)$ \\
Congenital lobar emphysema & $1(3.7)$ \\
Idiopathic & $6(21.4)$ \\
\hline
\end{tabular}

\section{DISCUSSION}

In the present study, an incidence of 1.5 per 1000 live births over an 8-year period was observed, with a median age at antenatal diagnosis being 26 weeks. In a cohort of 3137 stillbirths, NIH was diagnosed in $9 \%$ of the fetuses [5]. The difference from the present study could be due to the fact that only live born were included by us, whereas a large proportion of NIH probably die in utero.

Cardiovascular etiology $(n=5 ; 18.5 \%)$ was the most common etiology of NIH in the present study, which is similar to that observed in other studies [3]. Other significant causes include infectious (parvovirus, fetal toxoplasmosis, CMV, syphilis), hematological (alpha thalassemia), genetic (chromosomal abnormalities, skeletal dysplasias, metabolic causes like Gaucher, GM1 gangliosidosis, autosomal diseases like Noonan syndrome and placental causes (Twin-twin transfusion syndrome) [6]. Etiology could not be elucidated in $22 \%$ of cases, which is similar to the range of $15-25 \%$ reported in literature. Metabolic causes like lysosomal storage disorders may account for $29.6 \%$ of idiopathic NIH cases, if appropriate workup is done [7-9].

In a study from India reporting a 10 year experience of 33 cases, LSDs were observed to make up $22 \%$ of the etiology [10]. With the advent of newer diagnostic tools like next generation sequencing (NGS), it is possible to diagnose metabolic disorders causing NIH, like inborn errors of metabolism (IEM), which previously remained underdiagnosed. Retrospective study of amniotic fluid samples using hydrops fetalis (HydFet) panel making use of NGS led to significant improvement in diagnosis of IEM as a cause for NIH in a recent study [11].

Clinical outcome and survival rates depend largely on the underlying etiology. All neonates with cardiac and chylothorax as etiology survived while those with a suspected genetic syndrome died. In a large national database study, mortality rates were highest among neonates with congenital anomalies and lowest with congenital chylothorax [12]. Reported survival rates (excluding congenital anomalies) in NIH varies up to $31 \%-48 \%$ [13]. Overall survival rate in our cohort was $70 \%$ which was significantly higher than published survival rates. The possible explanation for the higher survival rates in our study could be strict protocol-based management and our preparedness in management, as majority of our cases were antenatally diagnosed.

Exclusion of stillbirths and fetal deaths was a limitation of our study. We could not confirm genetic diagnosis of the suspected syndromic cases. Moreover, the data was incomplete with respect to antenatal genetic work up done. 


\section{WHAT THIS STUDY ADDS?}

- We report on etiology, fetal interventions, postnatal management and outcomes of non-immune hydrops in neonates from India.

Non-immune hydrops fetalis is a rare clinical entity with varied etiology. Prognosis depends on the underlying etiology and chromosomal abnormalities generally tend to have a poor outcome. Efforts from multidisciplinary team including obstetricians, geneticists and neonatologists are required to achieve a favorable outcome.

Contributors: SM: writing the protocol, collecting data and having written the first draft of the manuscript; PA: helped in writing initial protocol, collection of data and contributed to the final manuscript; MJS: helped in interpretation of outcome measurement and contributed to final manuscript; AT: conceptualized the protocol, helped in writing protocol, and critically reviewed the final manuscript; AV,RA: helped in writing initial protocol and contributed to the final manuscript; AKD: gave critical inputs in final protocol and critically reviewed the manuscript.

Funding: None; Competing interests: None stated.

\section{REFERENCES}

1. Kenneth J. Moise Jr, Suzanne Lopez. Non immune Hydrops Fetalis. In: Cloherty and Stark's Manual of Neonatal Care. Eighth edition: Philadelphia, 2017.p.61-72.

2. Society for Maternal-Fetal Medicine (SMFM), Norton ME, Chauhan SP, Dashe JS. Society for Maternal-fetal Medicine (SMFM) Clinical Guideline \#7: Nonimmune Hydrops Fetalis. Am J Obstet Gynecol. 2015;212:127-39.

3. Bellini C, Hennekam RCM, Fulcheri E, Rutigliani M, Morcaldi G, Boccardo F, et al. Etiology of nonimmune hydrops fetalis: A systematic review. Am J Med Genet A. 2009;149A:844-51.

4. Bellini C, Hennekam RCM. Non-immune hydrops fetalis: A short review of etiology and pathophysiology. Am J Med
Genet Part A. 2012;158A:597-605.

5. McPherson E. Hydrops fetalis in a cohort of 3,137 still births and second trimester miscarriages. Am J Med Genet A. 2019; Sept 11 [Epub ahead of print].

6. Mascaretti RS, Falcão MC, Silva AM. Characterization of newborns with nonimmune hydrops fetalis admitted to a neonatal intensive care unit. Rev Hosp Clin Fac Med Sao Paulo. 2003;58:125-32.

7. Gimovsky AC, Luzi P, Berghella V. Lysosomal storage diseases as an etiology of non-immune hydrops: a systematic review. Am J Obstet Gynecol. 2015;212: 281-90.

8. Burin MG, Scholz AP, Gus R, Sanseverino MT, Fritish A, Magalhaes JA, et al. Investigation of lysosomal storage diseases in nonimmune hydrops fetalis. Prenat Diagn. 2004;24:653-7.

9. Ismail KM, Martin WL, Ghosh S, Whittle MJ, Kilby MD, et al. Etiology and outcome of hydrops fetalis. J Matern Fetal Med. 2001;10:175-81.

10. Sheth J, Mistri M. Lysosomal storage disorders in nonimmune hydrops fetalis (NIHF): An Indian Experience. JIMD Rep. 2017;35:47-52.

11. Sudrié-Arnaud B, Marguet F, Patrier S, Martinovic J, Louillet F, Broux F, et al. Metabolic causes of nonimmune hydrops fetalis: A next-generation sequencing panel as a first-line investigation. Clin Chim Acta. 2018;481:1-8.

12. Abrams ME, Meredith KS, Kinnard P, Clark RH. Hydrops fetalis: A retrospective review of cases reported to a large national database and identification of risk factors. Pediatrics. 2007;120:84-9.

13. Wilson RD, Baxter JK, Johnson MP, King M, Kaspersi S, Cromblehome TM, et al. Thoracoamniotic shunts: fetal treatment of pleural effusions and congenital cystic adeno- matoid malformations. Fetal Diagn Ther. 2004;19: 413-20. 